\title{
Worst-Case Residual Clipping Noise Power Model for Bit Loading in LACO-OFDM
}

\author{
Zhenyu Zhang*, Anas Chaaban ${ }^{\dagger}$, Chao Shen*, Hany Elgala ${ }^{\ddagger}$, Tien Khee $\mathrm{Ng}^{*}$, \\ Boon S. Ooi*, and Mohamed-Slim Alouini*
}

\author{
${ }^{*}$ CEMSE division, King Abdullah University of Science and Technology (KAUST), Thuwal 23955-6900, \\ Kingdom of Saudi Arabia. Email: zhenyu.zhang@kaust.edu.sa \\ ${ }^{\dagger}$ School of Engineering, University of British Columbia, Kelowna, BC V1V 1V7, Canada. Email: anas.chaaban@ubc.ca

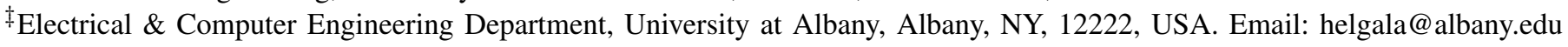

\begin{abstract}
Layered ACO-OFDM enjoys better spectral efficiency than ACO-OFDM, but its performance is challenged by residual clipping noise (RCN). In this paper, the power of RCN of LACO-OFDM is analyzed and modeled. As RCN is datadependent, the worst-case situation is considered. A worst-case indicator is defined for relating the power of $\mathrm{RCN}$ and the power of noise at the receiver, wherein a linear relation is shown to be a practical approximation. An LACO-OFDM bit-loading experiment is performed to examine the proposed RCN power model for data rates of 6 to $7 \mathrm{Gbps}$. The experiment's results show that accounting for RCN has two advantages. First, it leads to better bit loading and achieves up to $59 \%$ lower overall bit-error rate (BER) than when the $R C N$ is ignored. Second, it balances the BER across layers, which is a desired property from a channel coding perspective.
\end{abstract}

Index Terms-Bit loading, optical orthogonal frequency division multiplexing (O-OFDM), residual clipping noise, inter-layer interference.

\section{INTRODUCTION}

Along with the increasing demand for wireless communication throughput, researchers have been looking for methods to complement radio wireless transmission. Optical wireless communication (OWC) has emerged as a potential technology for coping with this demand in recent years [1]-[3]. In this context, intensity modulation and direct detection (IM/DD) has attracted great research attention [4], [5], along with optical orthogonal frequency division multiplexing (OFDM) as a means for providing fast and robust communication [6]. By arranging the subcarriers in a Hermitian symmetric way to obatin a real-valued signal and applying a direct current (DC) bias to complement negative values, DC-biased optical OFDM (DCO-OFDM) is obtained as a potential transmission technique for OWC. But DCO-OFDM is not energy efficient due to the DC bias. To avoid this energy inefficiency, asymmetrically clipped optical OFDM (ACO-OFDM) [7] is proposed, where data is loaded onto odd subcarriers only. Relying on the resulting anti-symmetric signal structure, ACO-OFDM produces a positive signal through clipping at zero, thus avoid the addition of a DC bias. However, as only half of the subcarriers are occupied, the spectral efficiency of ACO-OFDM is halved compared to DCO-OFDM. Balancing spectral and energy efficiency in optical OFDM is the focus of [8]-[10]. By combining ACO-OFDM signals in a layered structure, the techniques proposed in [8]-[10] can reach the same spectral efficiency as DCO-OFDM without requiring a DC bias. This technique is known as spectral and energy efficient OFDM (SEE-OFDM) [8], [9], or layered ACOOFDM (LACO-OFDM) [10]. Other optical-OFDM techniques have been studied in [11]-[13].

In this paper, we study LACO-OFDM. In this technique, each layer affects all the subsequent layers through clipping noise, so that without the correct demodulation of first layers (lower layers), demodulation of subsequent layers (higher layers) is not reliable. In the demodulation process, after demodulating lower layers, the clipping noise can be estimated and removed from higher layers. An incorrect estimation of clipping noise will cause interference to higher layers in the form of residual clipping noise (RCN), thus limiting their capacity. This problem has been addressed in the literature using noise cancellation [14] and diversity combining [15]. However, a power model of RCN is still not available, which prevents efficient optimization of LACO-OFDM performance.

In this paper, the RCN power of LACO-OFDM is analyzed and modeled by relating it to the power of noise at the receiver. This is motivated by the fact that $\mathrm{RCN}$ is caused by erroneous detection, which is caused by noise at the receiver. As detection errors in demodulation are dependent on transmitted data and noise realizations, to guarantee an independent performance, we consider the worst-case situation. We do this by modeling the relation between RCN power and noise power as linear, which turns out to be a practical approximation as demonstrated experimentally. An LACO-OFDM communication experiment with bit loading is performed to examine the performance of bit loading using the proposed RCN power model. The experiment shows that the proposed RCN power model can provide up to $59 \%$ performance improvement in the bit-error rate (BER) at data rates between 6 and $7 \mathrm{Gbps}$. Moreover, it allows to balance the BER of the layers, which otherwise increases from lower layers to higher layers due to RCN. This can be better from a channel coding perspective, since one could use the same 
code for all layers if the BER is balanced.

The rest of the paper is organized as follows. The IM/DD system and LACO-OFDM are introduced first in Sec. II. Then the worst-case-based RCN power model is proposed in Sec. III. Finally, an experiment is carried out to show the effectiveness of the proposed RCN power model in Sec. IV. We conclude in Sec. V.

\section{LACO-OFDM IN IM/DD SYSTEMS}

In this section, we describe the IM/DD system and LACOOFDM technique.

\section{A. The IM/DD OWC System}

In an IM/DD OWC system, the transmitter sends a nonnegative real signal $x(t)$ through a light source such as an LED or a laser diode (LD). This signal propagates over a wireless channel with impulse response $h(t)$. The receiver employs a photodetector (PD) to detect the signal, which is corrupted by noise. This noise includes thermal noise and background radiation and is considered Gaussian distributed, which is common in the OWC context [6]. The received signal can be expressed as $r(t)=\eta_{\mathrm{eo}} \eta_{\mathrm{oe}} h(t) \otimes x(t)+n(t)$, where $\otimes$ is the convolution operation, $\eta_{\mathrm{eo}}$ and $\eta_{\mathrm{oe}}$ are the electrical-to-optical $(\mathrm{E} / \mathrm{O})$ and optical-to-electrical $(\mathrm{O} / \mathrm{E})$ conversion efficiencies, which are assumed equal to 1 for simplicity. Thus, we obtain

$$
r(t)=h(t) \otimes x(t)+n(t) .
$$

The transmitted data rate are modulated into a signal $x(t)$ using methods such as LACO-OFDM which is the focus of this paper. This signal construction is explained next.

\section{B. LACO-OFDM Signal Construction}

Fig. 1 gives the block diagram of the modulation and demodulation of a $J$-layer LACO-OFDM IM/DD system [8]-[10]. In the transmitter, to generate a $J$-layer LACOOFDM signal, $\frac{N}{2}-1$ quadrature-amplitude modulation (QAM) symbols are generated from a data source, where $N=2^{J+1}$ which gives the highest spectrum efficiency of a $J$-layer LACO-OFDM. Those QAM symbols are arranged to a signal $X(k)$, with 'subcarriers' $k=0, \ldots, N-1$ according to a Hermitian asymmetric structure, so that the QAM symbols are assigned to $X(1), X(2), \ldots, X\left(\frac{N}{2}-1\right), X(0)=X\left(\frac{N}{2}\right)=0$, and $X(k)=X^{*}(N-k)$ for $k=1, \ldots, \frac{N}{2}-1$. Then, $X(k)$ is split into $J$ layers, $L_{1}, \ldots, L_{J}$, of the same length as $X(k)$, where the $j$-th layer has $X_{L_{j}}(k)=X_{L_{j}}^{*}(N-k)=X(k)$ for $k=2^{j-1}(2 i-1), i=1, \ldots, \frac{N}{2^{j+1}}$ and $X_{L_{j}}(k)=0$ otherwise. Then, after IFFT, clipping at zero, and converting to analog, we obtain the $j$-th layer signal $\bar{x}_{L_{j}}(t)$ which is the time-domain signal corresponding to $X_{L_{j}}(k)$. One block of LACO-OFDM is thus the sum of the time domain signals of all layers, i.e. $x(t)=\sum_{j} \bar{x}_{L_{j}}(t)$. Note that the Hermitian symmetry condition guarantees that $x_{L_{j}}(n)$ is real, and the clipping guarantees nonnegativity, which are necessary for IM/DD operation. The clipping of the $j$-th layer adds clipping noise only to layers $j+1, \ldots, J$ (see [8]-[10]).
At the receiver, $r(t)$ is first sampled in the 'A/D' block, then the channel response is equalized in the 'Equalizer' block, where we use channel inversion. This leads to

$$
y_{0}(n)=\bar{x}(n)+z(n),
$$

where $\bar{x}(n)=\sum_{j} \bar{x}_{L_{j}}(n)$, and $z(n)$ is the result of $n(t)$ passing through the 'A/D' and 'Equalizer' blocks. As the equalizer is linear, $z(n)$ is also Gaussian (possibly non-white). Then the frequency domain counterpart of $y_{0}(n)$ can be obtained through FFT, i.e.

$$
Y_{0}(k)=\bar{X}(k)+Z(k) .
$$

We write $\bar{X}(k)=\sum_{j} \bar{X}_{L_{j}}(k)$ and $Z(k)=\sum_{j} Z_{L_{j}}(k)$, where $\bar{X}_{L_{j}}(k)$ and $Z_{L_{j}}(k)$ respectively represent the $j$-th layer of $\bar{X}(k)$ and $Z(k)$. That is, $\bar{X}_{L_{j}}(k)=\bar{X}_{L_{j}}^{*}(N-k)=\bar{X}(k)$ and $Z_{L_{j}}(k)=Z_{L_{j}}^{*}(N-k)=Z(k)$ for $k=2^{j-1}(2 i-1)$, $i=1, \ldots, \frac{N}{2^{j+1}}$, and $\bar{X}_{L_{j}}(k)=Z_{L_{j}}(k)=0$ otherwise.

Then, the block labeled 'Layer 1' extracts

$$
Y_{L_{1}}(k)=\bar{X}_{L_{1}}(k)+Z_{L_{1}}(k) \text {. }
$$

Applying detection to $Y_{L_{1}}(k)$ in 'Det' block, the QAM symbols of the first layer can be obtained possibly with some errors, i.e.

$$
\hat{X}_{L_{1}}(k)=X_{L_{1}}(k)+\mathrm{E}^{(1)}(k),
$$

where $E^{(1)}(k) \stackrel{\text { IFFT }}{\longrightarrow} \varepsilon^{(1)}(n)$ corresponds to the detection error, and $\hat{X}_{L_{1}}(k)$ is the detection result of $X_{L_{1}}(k)$. This finishes the demodulation of the first layer. To demodulate the second layer, the clipping noise of the first layer should be removed first.

By applying IFFT to $\hat{X}_{L_{1}}(k)$, we obtain $\hat{x}_{L_{1}}(n)$, and then clipping $\hat{x}_{L_{1}}(n)$ at zero produces

$$
\bar{y}_{L_{1}}(n)=\frac{1}{2}\left(x_{L_{1}}(n)+\varepsilon^{(1)}(n)+\left|x_{L_{1}}(n)+\varepsilon^{(1)}(n)\right|\right) .
$$

By subtracting $\bar{y}_{L_{1}}(n)$ from $y_{0}(n)$, we obtain

$$
\begin{aligned}
y_{1}(n) & =y_{0}(n)-\bar{y}_{L_{1}}(n) \\
& =\sum_{j \neq 1} \bar{x}_{L_{j}}(n)+z(n)+n_{R C N}^{(1)}(n),
\end{aligned}
$$

where

$$
n_{R C N}^{(1)}(n)=\frac{1}{2}\left(\left|x_{L_{1}}(n)+\varepsilon^{(1)}(n)\right|-\left|x_{L_{1}}(n)\right|+\varepsilon^{(1)}(n)\right) .
$$

Here, the estimated signal of the first layer is removed from the received signal. Then, similar steps as the demodulation of the first layer can be repeated to demodulate the second layer and then higher layers, as shown in Fig. 1. In general, we can write

$$
y_{i}(n)=\sum_{j>i} \bar{x}_{L_{j}}(n)+z(n)+\sum_{j=1}^{i} n_{R C N}^{(i)}(n),
$$

where $n_{R C N}^{(i)}(n)$ is the RCN caused by the demodulation of the $i$-th layer. Note that if $\varepsilon^{(1)}(n)$ is zero, then $y_{1}(n)$ has no contribution from $x_{L_{1}}(n)$. Otherwise, $y_{1}(n)$ contains $\mathrm{RCN}$ 


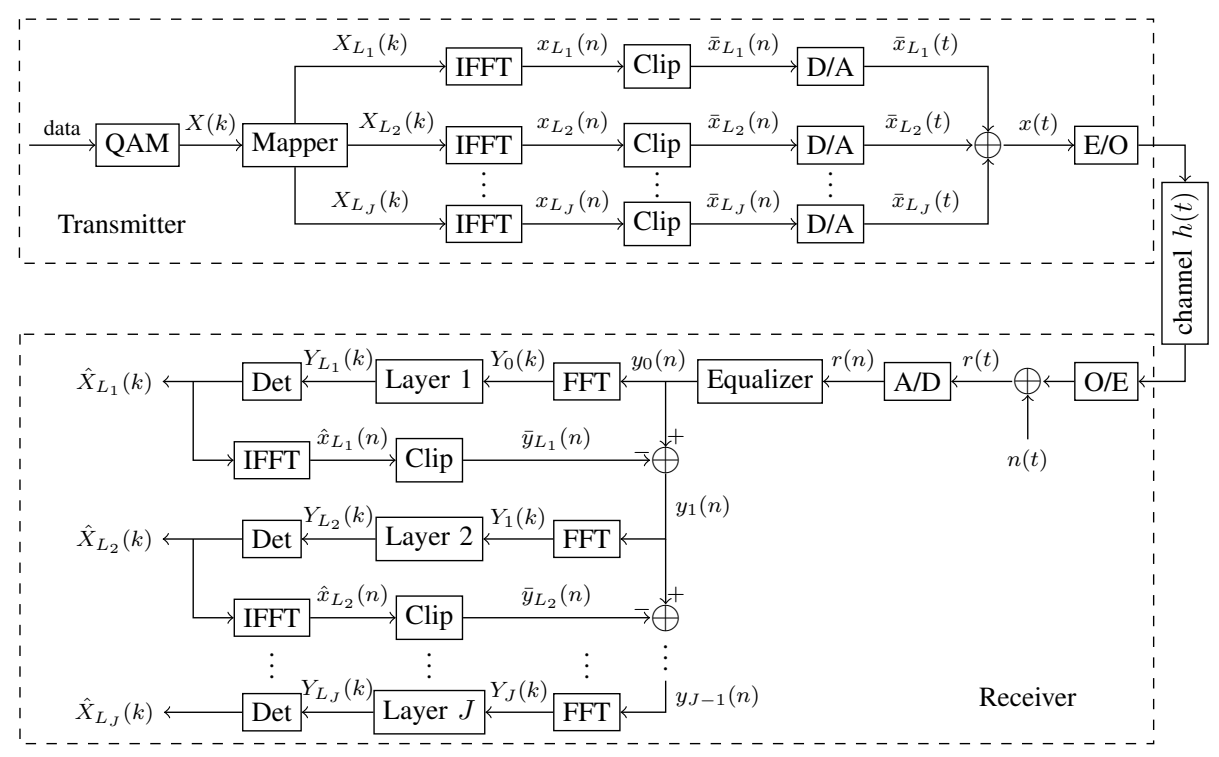

Fig. 1. Modulation and demodulation process of a $J$-layer LACO-OFDM IM/DD system.

which the receiver can not cancel, and which will disturb the demodulation of the second layer. Similarly for higher layers. Therefore, RCN should be considered as an additional noise source, and should be accounted for in the design and optimization of LACO-OFDM. In the following section, we focus on modeling the RCN.

\section{Worst-CASE RCN POWER MOdEL}

In this section, a RCN power model is proposed. Note that different data, including information data and noise realizations, will lead to different detection errors and hence different RCN. To avoid this complication, we discard the influence of different data through only focusing on the case which gives the highest RCN power, i.e. the worst case. Throughout this section, we write a signal $s(n) \stackrel{\mathrm{FFT}}{\longrightarrow} S(k)$ as the sum of its components on layers $1, \ldots, J$. That is $s_{L_{j}}(n) \stackrel{\text { FFT }}{\longrightarrow}$ $S_{L_{j}}(k)$ and $S_{L_{j}}(k)$ corresponds to the components of $S(k)$ on subcarriers of the $j$-th layer. And $\forall x(n), n=1,2, \ldots, N$, $P\{x\} \triangleq 1 / N \sum_{n=1}^{N}|x(n)|^{2}$.

Note that if noise power is relatively small, detection errors will be few, or vice versa. As a first step approximation, we assume a linear relation between the power of detection error and the power of noise. This is expressed as follows $P\left\{\varepsilon^{(1)}\right\}=\alpha^{(1)} P\left\{z_{L_{1}}\right\}$, where $\alpha^{(1)}>0$ is a variable which can be tuned to enhance the overall system performance. we call $\alpha^{(1)}$ the 'worst-case indicator',

As $\left|n_{R C N}^{(1)}(n)\right| \leq\left|\varepsilon^{(1)}(n)\right|$, then $P\left\{n_{R C N}^{(1)}\right\} \leq P\left\{\varepsilon^{(1)}\right\}$ with equality when all the power of detection error transfers to the power of $\mathrm{RCN}^{1}$. Thus, the worst-case $\mathrm{RCN}$ power after removing the first layer is

$$
P_{\mathrm{wRCN}}^{(1)}=P\left\{\varepsilon^{(1)}\right\}=\alpha^{(1)} P\left\{z_{L_{1}}\right\} .
$$

\footnotetext{
${ }^{1}$ This occurs e.g. if $x_{L_{1}}(n)+\varepsilon^{(1)}$ and $x_{L_{1}}(n)$ are positive (cf. (9)).
}

Then, we rewrite (8) as

$$
y_{1}^{\prime}(n)=\sum_{j \neq 1} \bar{x}_{L_{j}}(n)+z^{(1)}(n),
$$

where $z^{(1)}(n)=z(n)+\Delta^{(1)}(n)$ can be considered as a worst-case total noise. Here $\Delta^{(1)}(n)$ represent the worst-case RCN and $P\left\{\Delta^{(1)}\right\}=P\left\{\varepsilon^{(1)}\right\}$. Note that any guaranteed performance in (12) is also guaranteed in (8). This provides a model for the RCN produced from the first layer.

To extend this to higher layers, we can write

$$
y_{j}^{\prime}(n)=\sum_{i>j} \bar{x}_{L_{i}}(n)+z^{(j)}(n), \quad j=1, \ldots, J-1,
$$

where $z^{(j)}(n)$ is the worst-case total noise after removing the $j$-th layer (where the layers lower than $j$ are already removed) given by $z^{(j)}(n)=z^{(j-1)}(n)+\Delta^{(j)}(n)$, where $z^{(0)}(n)$ is used to denote $z(n)$ and $\Delta^{(j)}(n)$ is the worst-case RCN introduced from the $j$-th layer. Using this recursion, we can write

$$
z^{(j)}(n)=z(n)+\sum_{t=1}^{j} \Delta^{(t)}(n) .
$$

Similar to layer 1, the worst-case RCN power after removing the $j$-th layer can be written as

$$
P_{\mathrm{wRCN}}^{(j)}=P\left\{\Delta^{(j)}\right\}=\alpha^{(j)} P\left\{z_{L_{j}}^{(j-1)}\right\}
$$

for variable $\alpha^{(j)}$. At this step, it remains to express $P\left\{z_{L_{j}}^{(j-1)}\right\}$. Form (14), we obtain

$$
\begin{aligned}
P\left\{z_{L_{j}}^{(j-1)}\right\} & =P\left\{z_{L_{j}}+\sum_{t=1}^{j-1} \Delta_{L_{j}}^{(t)}\right\} \\
& =P\left\{z_{L_{j}}\right\}+\sum_{t=1}^{j-1} P\left\{\Delta_{L_{j}}^{(t)}\right\}+\beta_{j}
\end{aligned}
$$


where $\beta_{j}$ is resulted from possible correlation among $z_{L_{j}}(n)$ and $\Delta_{L_{j}}^{(t)}(n),(n=1, \ldots, j-1)$. In the experiment of Sec. IV, we set $\forall j, \beta_{j}=0$, which means $\Delta_{L_{j}}^{(t)}(n)(t=1, \ldots, j-1)$, $z_{L_{j}}(n)$ are assumed to be independent with each other and all have zero means. The effectiveness of this assumption is shown by the experimental results.

At this point, we assume that the power of $\Delta^{(t)}(n)$ is equally distributed over the subcarriers affected by $\Delta^{(t)}(n)$, i.e., those of layers $t+1, \ldots, J$. This will simplify analysis and will lead to good results as demonstrated by the experiment in Sec. IV. In this case, $P\left\{\Delta_{L_{j}}^{(t)}\right\}$ is proportional to the number of subcarriers in the $j$-th layer. As $\Delta^{(t)}(n)$ only affects layers $t+1, \ldots, J$, and the $j$-th layer $(j>t)$ has a fraction $2^{t-j}$ of the number of subcarriers affected by $\Delta^{(t)}(n)$, then

$$
P\left\{\Delta_{L_{j}}^{(t)}\right\}=\frac{1}{2^{j-t}} P\left\{\Delta^{(t)}\right\} .
$$

By substituting (16) and (18) in (15), we obtain

$$
P_{\mathrm{wRCN}}^{(j)}=\alpha^{(j)} P\left\{z_{L_{j}}\right\}+\sum_{t=1}^{j-1} \frac{\alpha^{(j)}}{2^{j-t}} P_{\mathrm{wRCN}}^{(t)}+\alpha^{(j)} \beta_{j} .
$$

This provides a recursion from which we can calculate $P_{\mathrm{wRCN}}^{(j)}$ starting with $j=1$ till $j=J$, based only on the noise power $P\left\{z_{L_{j}}\right\}$ which can be obtained from measurements. It can be seen from (19) that the worst-case RCN power after processing the $j$-th layer comes from two parts, the receiver noise at that layer corresponding to the first summand in (19), and the accumulated RCN from lower layers corresponding to the second and third summand.

The values of $\alpha^{(j)}$ can be chosen to maximize the performance. For the purpose of the experiment in the following section, we fix $\alpha^{(j)}=\alpha$ for all $j$, and the value of $\alpha$ is varied to investigate its impact on performance.

\section{EXPERIMENT}

To investigate the effect of $\mathrm{RCN}$ on the performance of LACO-OFDM and verify the effectiveness of the proposed RCN power model, we use an experimental approach. A bit-loading LACO-OFDM communication experiment is performed to compare the performance of the bit loading results with and without the RCN power model. The experimental set-up is introduced first, and then the procedure is described step by step, and the results of each step are presented and discussed.

\section{A. Experimental Set-Up}

Fig. 2 and Fig. 3 shows the block diagram and pictures of the experimental setup. In this experiment, we generate the transmit signal using a computer. This signal is then loaded to a Tektronix AWG70002A Arbitray Waveform Generator (AWG) to generate the continuous signal waveform. The output peak-to-peak voltage (Vpp) is set at $500 \mathrm{mV}$. The output of AWG is then coupled, using a Mini-circuits ZFBT-4R2GWFT+ bias-T, with a direct current (DC) of $100 \mathrm{~mA}$ generated by a current source. The DC is used to provide a stable

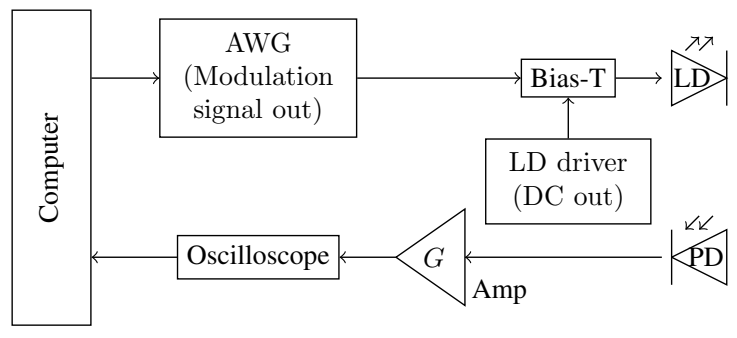

Fig. 2. Block diagram of the experiment set-up.

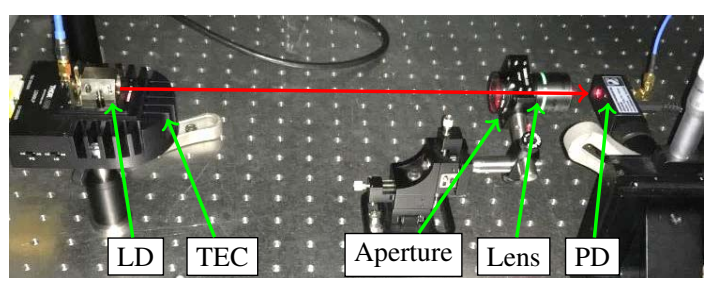

Fig. 3. Experiment set-up of experiment 2. The red arrow traces the light beam from the LD to the PD.

operating point for the LD. The resulting signal is injected into a Mitsubishi ML101U29 device, which is a $400 \mathrm{~mW}$ $660 \mathrm{~nm}$ red LD. To avoid the effects of temperature change on the performance of the LD, we mount LD in the Thorlab LDM9LP thermoelectric cooler (TEC), where the temperature is set at $18^{\circ} \mathrm{C}$. The LD emits towards an Alphalas UPD-50-UP photodetector (PD) at a distance of $30 \mathrm{~cm}$ through a focusing lens. Since the maximum input optical power of this PD is $10 \mathrm{~mW}$ while the used LD can emit around $400 \mathrm{~mW}$, a small aperture is placed right before the lens in order to reduce the light intensity for the safety of the PD. And a PSPL5866 high speed amplifier which can provide around $25 \mathrm{~dB}$ linear gain is adopted to amplify the output of the PD. The amplified signal is recorded using an oscilloscope and then sent back to the computer for processing.

\section{B. Experimental Procedure and Results}

Within the introduced set-up, a 7-layer LACO-OFDM system is adopted. The bandwidth of the LACO-OFDM signal is set to $1 \mathrm{GHz}$. This signal is upsampled by a factor of 8 so that each block consists of 2048 samples, leading to a bandwidth of $8 \mathrm{GHz} .^{2}$ The sampling rate of the AWG is configured at $16 \mathrm{GS} / \mathrm{s}$ accordingly to match the signal bandwidth. Then, the bit-loading LACO-OFDM communication experiments are performed in four steps as follows.

1) Noise Power and Channel Estimation: The noise power spectral density is measured by setting the LACO-OFDM signal to zero and driving the LD using the $100 \mathrm{~mA}$ DC bias only. Then, hundreds of measurements are recorded, and the noise power spectral density is averaged leading to Fig.4(a).

The channel gain in Fig. 4(b) is estimated by sending random quadratic-phase-shift-keying (QPSK) symbols with constant energy using LACO-OFDM. [16]

\footnotetext{
${ }^{2}$ For the purpose of this experiment, this is done using a 2048-point FFT, where the LACO-OFDM signal occupies only 256 points thereof.
} 


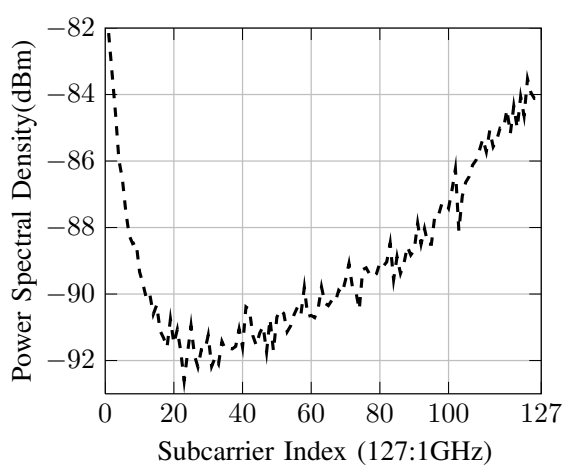

(a) Noise power spectral density.

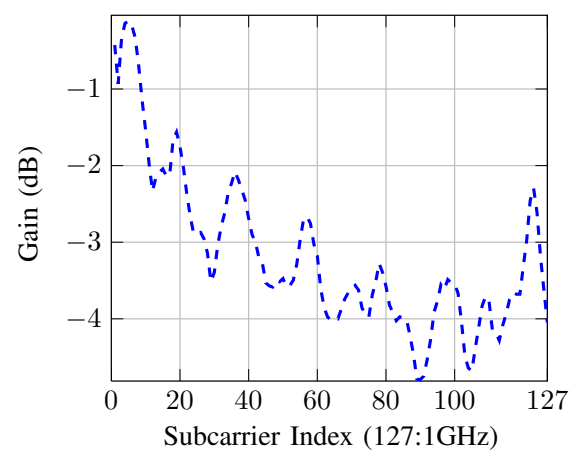

(b) Channel gain.

Fig. 4. Channel estimation results in experiment 1. (a) Noise power spectral density, (b) channel gain.
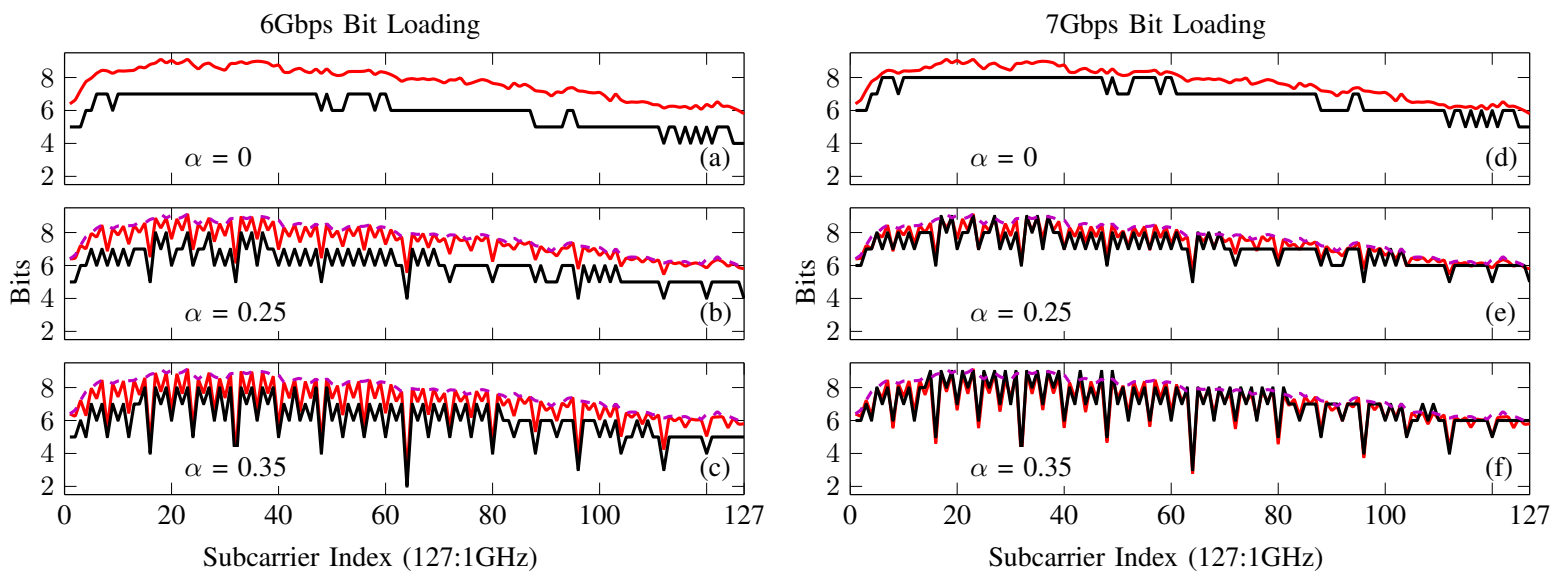

Fig. 5. Bit loading Results of $6 \mathrm{Gbps}$ and $7 \mathrm{Gbps}$ under $\alpha=0,0.25,0.35$. (a)-(c) target $6 \mathrm{Gbps}$, and (d)-(f) target 7Gbps. (The top solid line of each subfigure is the estimated theoretical capacity with RCN in consideration and the bottom solid line is the bit loading results. Dashed line in (b)(c)(e)(f) is the theoretical capacity estimated with $\alpha=0$.

2) Capacity Estimation with/without RCN: This step is performed in order to get an estimate about the capacity of each subcarrier, which will be used for bit loading in step 3. The noise power obtained in step 1 is used to estimate the power of RCN according to (19) where we set $\forall j, \beta_{j}=0$, and $\alpha$ is varied among $0,0.25$, and 0.35 . Note that $\alpha=0$ implies that RCN is zero, i.e., not taken into account. Then, the received signal-to-noise-ratio (SNR) under different $\alpha$ of each subcarrier can be obtained easily. Note that the transmitted constellations of each subcarrier has the same scale although their size (i.e. number of symbols) are different. Finally, we calculate the capacity of each subcarrier by substituting the obtained SNR to Shannon's capacity expression $\log _{2}(1+\mathrm{SNR})$. This will be used as a benchmark for bit loading in the next step.

3) Bit Loading: After obtaining the estimated capacity in step 2, bit loading can be performed based on it. This is done as follows. First, we set a target data rate, and we set the number of bits on all subcarriers to zero. Then, bits are loaded one by one to the subcarrier which has the largest capacity margin, i.e., gap between estimated capacity and loaded bits. This is repeated until the target data rate is reached. In this experiment, the target data rate is varied from 6 to $7 \mathrm{Gbps}$.

Fig. 5 shows the obtained bit loading with target data rates of 6 and $7 \mathrm{Gbps}$ under $\alpha \in\{0,0.25,0.35\}$. In each subfigure, the top solid line is the estimated theoretical capacity, the bottom solid line is the bit loading results. It can be seen that larger $\alpha$ reduces the number of loaded bits in higher layers. In order to reach the target rate, subcarriers of lower layers are loaded with more bits (compare subcarriers $60-80$ in the three $\alpha$ cases).

4) BER Performance: The last step is using the experimental set-up to measure the BER performance under the bit loading scheme obtained in step 3. Fig. 6 shows the results. The impact of $\alpha$ on the BER performance can be observed in this figure. Setting $\alpha=0$ ignores RCN, which results in some subcarriers being loaded with more bits than what they are able to deliver. Increasing $\alpha$ to 0.25 improves the BER by a clear margin with respect to $\alpha=0$. This is because bit loading in this case becomes aware of RCN, and does not overload subcarriers. Increasing $\alpha$ further to 0.35 in this experiment makes performance worse. This is because $\alpha=0.35$ overestimates RCN which leads to overloading lower layers. The improvement between $\alpha=0$ (RCN agnostic) and $\alpha=0.25$ 


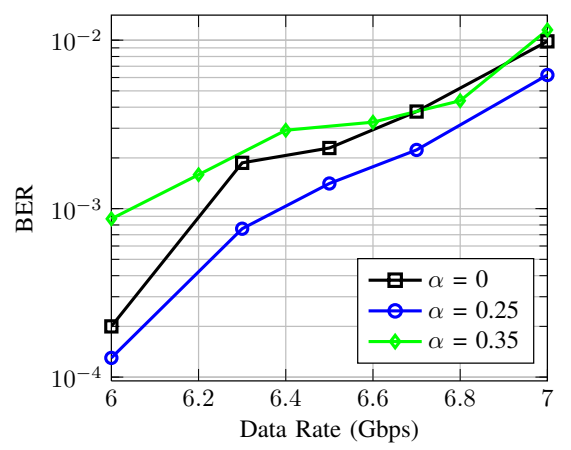

Fig. 6. BER v.s. data rate under $\alpha=0,0.25,0.35$.

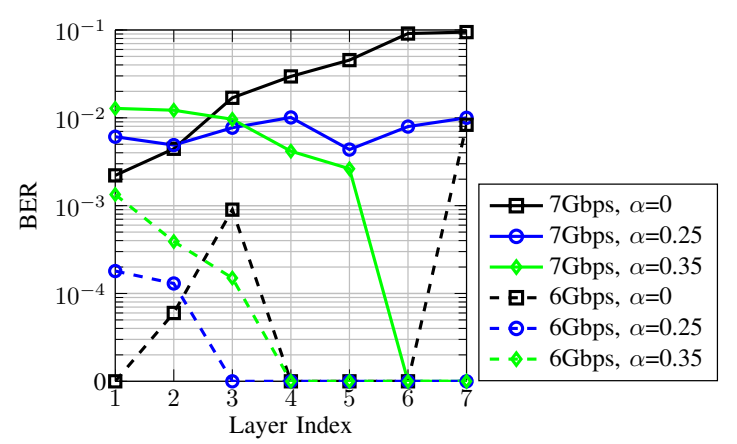

Fig. 7. BER of each layer when data rate is $6 \mathrm{Gpbs}$ and $7 \mathrm{Gbps}$, under $\alpha=0,0.25,0.35$.

( $\mathrm{RCN}$ aware) is as much as $59 \%$ at a target rate $6.3 \mathrm{Gbps}$ and $37 \%$ at a target rate of $7 \mathrm{Gbps}$.

In order to see the impact of $\alpha$ on the BER across layers of LACO-OFDM, we plot the BER versus the layer index $j=1, \ldots, 7$ in Fig. 7, for rates of 6 and $7 \mathrm{Gbps}$. It can be seen that, for both rates, $\alpha=0$ is better than $\alpha=0.25$ or 0.35 in the first two layers, and worse (or not better) in the last five layers. On the other hand, $\alpha=0.35$ is the worse in the first two layers and the best in the last four layers. The case $\alpha=0.25$ shows an 'equalized' BER over all layers, i.e., the BER of all layers is roughly the same, with a BER between the $\alpha=0$ and $\alpha=0.35$ cases. The lowest overall BER and the balanced BER of layers make $\alpha=0.25$ appropriate as a worst-case indicator for rates between 6 and $7 \mathrm{Gbps}$.

\section{CONCLUSION}

In this paper, the residual clipping noise (RCN) of LACOOFDM is analyzed and its power is modeled. The proposed RCN power model is examined in a bit loading experiment. The experiment shows that bit loading using the proposed RCN power model can avoid overloading subcarriers and result in a better overall performance. The improvement has been demonstrated for rates between $6 \mathrm{Gbps}$ and $7 \mathrm{Gbps}$, and this BER improvement can reach up to $59 \%$. Moreover, this model allows us to balance the BER across the layers of LACO-OFDM, which is a desired property from channel coding perspective. Note that since RCN exists also in other layered optical OFDM schemes, such as ADO-OFDM [11],
HACO-OFDM [12], and ASCO-OFDM [13], the proposed model can be useful for those schemes as well.

In this work, we used an equal worst-case indicator across layers. This might be not ideal, but can be beneficial in practice. Note that this indicator might depend on the data rate. For example, if the data rate is far less than the channel capacity, the detection error probability will be low, and the worst-case indicator should be close to zero. As the data rate increases towards the channel capacity, the detection errors probability increases, and this indicator should increase. Optimizing this indicator is an interesting investigation for future works.

\section{REFERENCES}

[1] T. Nagatsuma, G. Ducournau, C. C. Renaud. "Advances in terahertz communications accelerated by photonics," Nature Photon., vol. 10, no. 7, pp. 371-379, 2016.

[2] M. A. Khalighi, M. Uysal. "Survey on free space optical communication: A communication theory perspective," IEEE Commun. Surveys Tuts., vol. 16, no. 4, pp. 2231-2258, 2014

[3] H. Burchardt, N. Serafimovski, D. Tsonev, et al. "VLC: Beyond pointto-point communication," IEEE Commun. Mag., vol. 52, no. 7, pp. 98$105,2014$.

[4] A. Chaaban, Z. Rezki, M. S. Alouini. "On the capacity of the intensitymodulation direct-detection optical broadcast channel," IEEE Trans. Wireless Commun., vol. 15, no. 5, pp. 3114-3130, 2016.

[5] T. Y. Elganimi. "Performance comparison between OOK, PPM and pam modulation schemes for free space optical (FSO) communication systems: analytical study," Int. J. Comput. Appl., vol 79, no. 11, pp. 2227, 2013.

[6] S. Arnon, J. Barry, G. Karagiannidis, R. Schober, and M. Uysal, Eds. Advanced Optical Wireless Communication Systems. Cambridge, U.K.: Cambridge Univ. Press, 2012.

[7] J. Armstrong, B. J. C. Schmidt, D. Kalra, H. A. Suraweera, and A. J. Lowery, "Performance of asymmetrically clipped optical OFDM in AWGN for an intensity modulated direct detection system," in Proc. IEEE GLOBECOM, 2006, pp. 1-5.

[8] H. Elgala and T. D. C. Little, "SEE-OFDM: Spectral and energy efficient OFDM for optical IM/DD systems," in Proc. IEEE Int. Symp. Pers., Indoor, and Mobile Radio Commun. (PIMRC), Sep. 2014, pp. 851-855.

[9] E. Lam, S. K. Wilson, H. Elgala, and T. D. C. Little, "Spectrally and energy efficient OFDM (SEE-OFDM) for intensity modulated optical wireless systems," arXiv preprint, arXiv:1510.08172, 2015.

[10] Q. Wang, C. Qian, X. Guo, Z. Wang, D. G. Cunningham, and I. H. White, "Layered ACO-OFDM for intensity-modulated direct-detection optical wireless transmission," Opt. Express, vol. 23, no. 9, pp. $12382-$ 12393, May 2015.

[11] S. Dissanayake and J. Armstrong, "Comparison of ACO-OFDM, DCOOFDM and ADO-OFDM in IM/DD systems," J. Lightw. Technol., vol. 31, no. 7, pp. 1063-1072, Apr. 2013

[12] B. Ranjha and M. Kavehrad, "Hybrid asymmetrically clipped OFDMbased IM/DD optical wireless system," IEEE/OSA J. Opt. Commun. Netw., vol. 6, no. 4, pp. 387-396, Apr. 2014.

[13] N. Wu and Y. Bar-Ness, "A novel power-efficient scheme asymmetrically and symmetrically clipping optical (ASCO)- OFDM for IM/DD optical systems," EURASIP J. Advances Signal Process., vol. 3, Jan. 2015.

[14] Q. Wang, Z. Wang, X. Guo, et al. "Improved receiver design for layered ACO-OFDM in optical wireless communications," IEEE Photon. Technol. Lett., vol. 28, no. 32016, pp. 319-322, Feb. 2016.

[15] M. M. A. Mohammed, C. He, and J. Armstrong, "Diversity Combining in Layered Asymmetrically Clipped Optical OFDM," J. Lightwave Technol. vol. 35, no. 11, pp. 2078-2085, Jun. 2017.

[16] D. Tsonev, H. Chun, S. Rajbhandari, et al. "A 3-Gb/s single-LED OFDM-based wireless VLC link using a gallium nitride LED". in IEEE Photon. Technol. Lett., vol. 26, no. 7, pp. 637-640, Apr. 2014. 